\title{
Repeated Cocaine Self-Administration Alters Processing of Cocaine-Related Information in Rat Prefrontal Cortex
}

\author{
WenLin Sun and George V. Rebec \\ Program in Neuroscience, Department of Psychological and Brain Sciences, Indiana University, Bloomington, Indiana 47405-7007
}

\begin{abstract}
One of the core symptoms of cocaine addiction is compulsive drug-seeking behavior. Although the precise neural substrates are unknown, it has been hypothesized that this behavior involves cocaine-induced hypofunction of the prefrontal cortex (PFC) or "hypofrontality." To test this hypothesis, PFC neuronal activity was monitored in rats during $\sim 3$ weeks of cocaine self-administration (SA). Rats were trained to press a lever to self-administer cocaine in daily $2 \mathrm{~h}$ sessions. Responding was reinforced contingent on a modified fixed-ratio 5 schedule of reinforcement. In the first SA session, the overall firing rate and burst rate were significantly decreased after cocaine infusions relative to the period immediately before the session. These effects disappeared after $\geq 10 \mathrm{~d}$ of drug SA and were replaced by a significant increase in burst duration and firing rate within a burst. Notably, however, the level of basal activity before the first drug infusion of each SA session decreased significantly after multiple weeks of cocaine exposure. Collectively, these data support the view that although repeated sessions of cocaine SA decrease basal PFC activity, increased burst-related firing in response to cocaine infusions suggests that processing of cocaine-related information is enhanced and may contribute to increased control by cocaine over cocaine-seeking behavior.
\end{abstract}

Key words: cocaine; prefrontal cortex; electrophysiology; self-administration; addiction; neuroplasticity

\section{Introduction}

Relapse is one of the most challenging issues in treatment of cocaine addiction. Understanding its neurobiological mechanisms is a critical step toward development of better antiaddiction therapies. A recent theory proposes that drug-induced hypofunction of the prefrontal cortex (PFC) may be critically involved in compulsive drug-seeking and drug-taking behavior (Jentsch and Taylor, 1999). It is well known that the PFC plays an important role in control of behavior by regulating the function of subcortical motor structures such as the basal ganglia (Roberts and Wallis, 2000). For example, lesions to monkey PFC impair reversal learning of object or spatial discrimination tasks (Dias et al., 1996). Such effects are thought to reflect an inability to inhibit responding toward the stimuli previously paired with a reward (Roberts and Wallis, 2000). Chronic exposure to cocaine mimics these lesion effects. Cocaine-treated rats, for example, show persistent responding to the stimulus previously associated with cocaine and do not easily shift their responding to the stimuli currently associated with reward (Jentsch et al., 2002). This biased and persistent responding is reminiscent of the focused and persistent drug-seeking behavior observed in drug addicts. Indeed, recent imaging studies reveal that the metabolic activity of the PFC in drug addicts is lower compared with normal controls

\footnotetext{
Received April 3, 2006; revised June 5, 2006; accepted June 23, 2006.

This work was supported by National Institutes of Health Grant DA02451. We thank Paul Langley and Faye Caylor for technical and administrative support.

Correspondence should be addressed to Dr. George V. Rebec, Program in Neuroscience, Department of Psychological and Brain Sciences, Indiana University, 1101 East 10th Street, Bloomington, IN 47405-7007. E-mail: rebec@indiana.edu.

DOI:10.1523/JNEUROSCI.1413-06.2006

Copyright $\odot 2006$ Society for Neuroscience $\quad 0270-6474 / 06 / 268004-05 \$ 15.00 / 0$
}

(Volkow et al., 2003). Interestingly, exposure of addicts to environmental stimuli previously associated with the drug experience activates the PFC, which is correlated with self-reported drug craving (Childress et al., 1999). Rats self-administering cocaine also show cue-related activation of PFC neurons (Rebec and Sun, 2005). It has been suggested that lower basal PFC activity may amplify the PFC response to drugs and drug conditioned stimuli (CSs) and therefore allow these stimuli to exert greater control over behavior.

Imaging studies, however, cannot determine whether "hypofrontality" is a condition induced by drugs of abuse or exists as a pre-addiction condition. To determine whether chronic exposure to cocaine can induce hypofunction of the PFC, we used chronically implanted microwire electrodes to record neuronal activity in rat PFC during a 3 week period of cocaine selfadministration (SA). It also was of interest to investigate whether chronic exposure to cocaine increases PFC neuronal responding to cocaine, because such increases may underlie enhanced processing of cocaine-related information in the PFC. Our results show that although chronic cocaine SA indeed decreased basal PFC neuronal activity, PFC neuronal responding to cocaine was increased as evidenced by increased burst duration and firing rate within a burst, suggesting that processing cocaine-related information is enhanced in rats with a history of cocaine SA.

\section{Materials and Methods}

Subjects. Male Sprague Dawley rats $(350-400$ g) were used. One week before operant training, rats were placed on a restricted diet to reach $\sim 85 \%$ of free-feeding weight. After training, ad libitum access to food was available for 1 week before and after surgery. Food restriction was then reinstated to maintain $85-90 \%$ of free-feeding weight throughout 
the experiments. Water was available ad libitum all the time. Lights were on for $12 \mathrm{~h}$ beginning at 7:30 A.M. The experiments were conducted in the light cycle (between 9:00 A.M. and 6:00 P.M.). All procedures were approved by the Indiana University Animal Care and Use Committee.

Operant chambers. Two locally constructed Plexiglas operant chambers, equipped with one Plexiglas lever and two cue lights mounted on a side wall, were used. A fluid well was installed below the lever. A house light was installed on the other side wall. An audio speaker was located outside the chamber. Each chamber was housed inside a grounded galvanized cubicle to shield electric noise. A fluid pump with adjustable speeds (PHM-100VS; Med Associates, East Fairfield, VT) was placed outside the cubicle. A modified electrical commutator/fluid swivel assembly (Plastics One, Roanoke, VA) was mounted on the ceiling of the cubicle to allow uninterrupted flow of electrical signals and intravenous drug solution.

Operant training. Rats $(n=9)$ first learned to press the lever reinforced by sucrose solution $(10 \%, \mathrm{v} / \mathrm{v})$ under a fixed-ratio 1 (FR1) schedule in a daily 30 min session. Once rats obtained 60 reinforcements within 30 min, the reinforcement schedule increased to FR 3 and then to FR5. Rats were then scheduled for surgery.

Surgical procedures. Animals received ketamine ( $80 \mathrm{mg} / \mathrm{kg}$, i.p.) and xylazine $(10 \mathrm{mg} / \mathrm{kg}$, i.p.) for anesthesia. Ketamine supplements $(10 \mathrm{mg} /$ $0.1 \mathrm{ml}$ ) were given as needed throughout surgery. A catheter made from PE10 and PE50 tubing was inserted into the right jugular vein as described previously (Sun and Rebec, 2003). After catheterization, rats were fixed in a stereotaxic frame for implantation of microwire electrodes as described previously (Rebec and Sun, 2005). Each electrode assembly consisted of eight $25-\mu \mathrm{m}$-diameter Fromvar-insulated stainless steel microwires (California FineWire Company, Grover Beach, CA) arranged in cylindrical bundles and threaded through a stainless steel hypodermic needle (27 ga). The electrodes were implanted bilaterally in the PFC at an angle of $10^{\circ}$ away from the midline. The coordinates for the PFC were as follows: $+3.0 \mathrm{~mm}$ anteroposterior, $\pm 0.6 \mathrm{~mm}$ mediolateral, and -3.0 $\mathrm{mm}$ dorsoventral, relative to the bregma, midline, and skull surface, respectively. Four additional holes were drilled for stainless steel support screws. Dental acrylic held the screws and electrodes in place. During recovery, animals received daily $0.1 \mathrm{ml}$ of gentamicin $(10 \mathrm{mg} / \mathrm{ml}$, i.v.) for 1 week to minimize infection.

SA training. We used a modified FR5 schedule in which the first response was reinforced by an infusion of $0.125 \mathrm{mg}$ of cocaine in a volume of $0.05 \mathrm{ml}$ over $1 \mathrm{~s}$. Compound stimuli (cue light and tone) were paired with cocaine infusions and lasted $4 \mathrm{~s}$, followed by a $16 \mathrm{~s}$ timeout signaled by illumination of the house light. During the cocaine infusion and timeout (total $20 \mathrm{~s}$ ), responding was recorded but had no programmed consequences. After the first cocaine infusion, subsequent responses were reinforced contingent on an FR5 schedule. The session ended when $2 \mathrm{~h}$ passed or 60 infusions were delivered, whichever occurred first. Catheter patency was evaluated as necessary by infusing $0.1 \mathrm{ml}$ of Brevital (1\%); loss of muscle tone within $5 \mathrm{~s}$ after infusion indicated a patent catheter.

Electrophysiological recording. A headset attached to a flexible wire cable was plugged into the electrode assembly, and the fluid tubing was connected to the guide cannula installed on the rat's head during surgery. The headset contained 16 miniature, unity-gain, and field-effect transistors. The cable was connected to the commutator/swivel assembly. Electrical signals from the microwires were passed through a pre-amplifier and fed into the SIG board of the Multichannel Acquisition Processor System (MAPS) (Plexon, Dallas, TX) in which the signals were further amplified, filtered $(0.3-10 \mathrm{kHz})$, and digitized $(40 \mathrm{kHz}$ analog-to-digital conversion). Spike sorting was performed by the digital signal processor board on the MAPS controlled by the Sort Client program (Plexon) in real time. The first step was to set a voltage threshold for each channel. Typically, the threshold was set to reach a minimum signal-to-noise ratio $>2.5$ :1. A template algorithm was used to isolate units (Rebec and Sun, 2005). All of the waveforms that matched the templates were saved for additional off-line spike sorting (Plexon), which was used to refine the discrimination of the units. Isolation of units was verified by post hoc analysis of interspike intervals (ISIs) (Gulley et al., 2004). The interspikeinterval histograms of single units must exhibit a clearly recognizable refractory period $(>1 \mathrm{~ms})$. When all units were isolated $(\sim 30 \mathrm{~min})$, baseline activity was recorded for $1 \mathrm{~min}$, after which the session started. Recording lasted the entire session. Spike waveforms and time stamps were saved on the computer.

The events occurring in the operant chamber including lever press, onset of the stimuli, and fluid pumps were recorded and controlled by a program (Med Associates) running on a Pentium computer. These events were sent to the digital inputs on the MAPS for time stamping through a transistor-transistor logic board controlled by the program.

Histology. After the experiments, rats were anesthetized in preparation for subsequent histological analysis. A $10 \mathrm{~s}, 20 \mu \mathrm{A}$ positive current was passed across all electrodes to mark recording sites. Rats were then perfused transcardially with PBS $(0.9 \%)$, followed by $4 \%$ paraformaldehyde $/ 2 \%$ potassium ferrocyanide, which reacts with iron deposits to produce Prussian blue spots at the electrode tip. All electrodes were marked to verify that they were within the target area; individual recording sites, however, did not have enough spatial resolution to be identified reliably. To ensure that all marked wires were identified, tissue sections $(60 \mu \mathrm{m}$ thickness) were analyzed up to $2 \mathrm{~mm}$ anterior and posterior to the electrode entry point.

Statistics. Data from sessions 1, 10-12, and 19-21 were used for statistical analysis. The firing rate and burst rate were calculated as spikes per second and bursts per minute, respectively. Baseline data were based on a $1 \mathrm{~min}$ period immediately before the session. The firing rate in response to self-administered cocaine was based on a $10 \mathrm{~s}$ time window starting from $10 \mathrm{~s}$ after each cocaine infusion and averaged across all infusions in a session. This time window was selected because the rats showed a sudden increase in locomotion and a burst of face washing during this period, which collectively suggest the onset of direct pharmacological effects of cocaine. Because we previously showed that PFC neurons are phasically activated by cocaine CSs with a latency of $\sim 500 \mathrm{~ms}$ and a duration of $\sim 700 \mathrm{~ms}$ (Rebec and Sun, 2005), analyses that include the entire timeout period would be confounded by neuronal responding to CSs. In addition, PFC neurons do not respond to onset of the house light in the presence of the CSs paired with cocaine infusions (Rebec and Sun, 2005). Given these considerations, we believe that any change in neuronal activity during the $10 \mathrm{~s}$ time window is attributable to the effects of cocaine rather than those of the CSs or house light. We also analyzed burst firing by assessing the burst rate, the rate within a burst, and burst duration. Bursts were defined as a train of at least three spikes having an $80 \mathrm{~ms}$ maximum onset ISI and a $160 \mathrm{~ms}$ minimum offset ISI. There is not a universal standard for defining bursts, and different investigators have used different ISIs as cutoff points to define burst activity recorded from neurons in the PFC and hippocampus neurons (Legendy et al., 1985; Quirk et al., 1999; Laviolette et al., 2005). To determine whether analyses with different cutoff ISIs produce different statistical results, we analyzed the data using our parameters and the cutoff ISI $45 \mathrm{~ms}$ proposed by Grace's group (Laviolette et al., 2005). No differences were found, except that the analysis with our parameters revealed a significant decrease in burst rate by cocaine in the first cocaine SA session. Thus, all results were reported based on our parameters. Because all of these electrophysiological variables did not follow Gaussian distribution (D'Agostino and Pearson omnibus normality test, $p<0.01$ ), a nonparametric Kruskal-Wallis test was used to compare their median rather than mean. Differences in neuronal activity across sessions were compared with Dunn's test. Differences between baseline activity and activity after cocaine infusions within each session were tested with a Wilcoxon signed-rank test. In contrast, the amount of cocaine infused in different sessions passed the normality test, and therefore a repeated one-way ANOVA was used to compare cocaine intake. The significance level was set at $\alpha=0.05$.

\section{Results}

The positions of our microwire electrodes are shown schematically in Figure 1. All recording sites were located within area 1 of the anterior cingulate and dorsal prelimbic cortex.

The amount of cocaine infused during sessions $1,10-12$, and 19-21 was $2.1 \pm 0.5,4.0 \pm 0.4$, and $2.6 \pm 0.5 \mathrm{mg} / \mathrm{session}$, respectively. Because two rats did not reach the third week of training because of catheter failure, the ANOVA was based on the remain- 

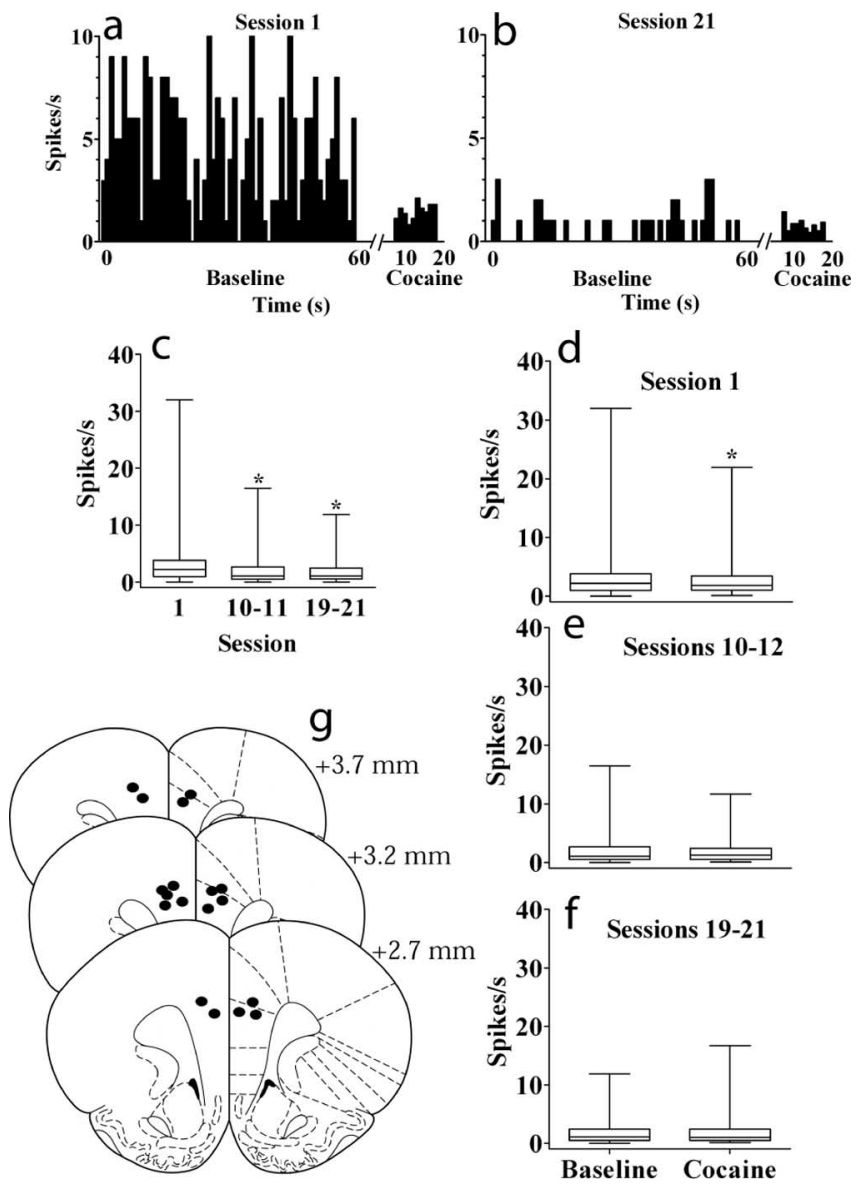

Figure 1. Effects of cocaine on the firing rate of PFC neurons. Data are presented as median with the 25th and 75th percentiles in the box-and-whisker graphs. The box extends from the 25th percentile to the 75 th percentile with the line at the median (the 50th percentile). Whiskers extend below and above the box to show the lowest and highest values. $\boldsymbol{a}, \boldsymbol{b}$, Examples of rate histograms (1 ms bin) from a representative neuron recorded in sessions 1 and 21. $c$, Basal rate for the sessions indicated. ${ }^{*} p<0.001$, significantly different from the basal rate recorded from session 1 (Kruskal-Wallis test). $\boldsymbol{d}-\boldsymbol{f}$, Rate during cocaine SA. Cocaine significantly ( $p<$ 0.05 ) decreased the firing rate during session 1 but not the other sessions. $\boldsymbol{g}$, Schematic depiction of recording sites in the PFC. The filled circles represent the locations of microwire bundles. All recording sites were located in area 1 of the cingulate or dorsal prelimbic cortex. Coronal brain section images were adapted from the atlas of Paxinos and Watson (1998).

ing seven rats. Although there was an increase in the amount of cocaine infused during sessions $10-12$, this increase was not significant $\left(F_{(2,12)}=3.88 ; p>0.05\right)$.

Nine rats were recorded in session 1 and sessions $10-12$ and yielded 132 and 95 units, respectively. Seven rats were recorded in sessions 19-21 and yielded 79 units. As shown in Figure 1, the median (50th percentile range) basal firing rate of PFC neurons recorded in sessions $1,10-12$, and $19-21$ were $2.2(0.9-3.8)$ spikes/s, 1.1 (0.5-2.6) spikes/s, and 1.0 (0.5-2.4) spikes/s, respectively. The basal firing rates during sessions $10-12$ and 19-21 were significantly decreased compared with session 1 (KruskalWallis test, $p<0.001$ ). There were no significant differences between sessions 10-12 and 19-21. In addition, we investigated whether these neurons show changes in responding to cocaine. We found that the firing rate was significantly decreased after cocaine infusions during the first SA session (Wilcoxon signedrank test, $p<0.05$ ) but not during the other sessions.

Next, we investigated the effects of chronic cocaine SA on basal burst activity of PFC neurons. As shown in Figure 2, the median basal burst rate (50th percentile range) in sessions 10-12
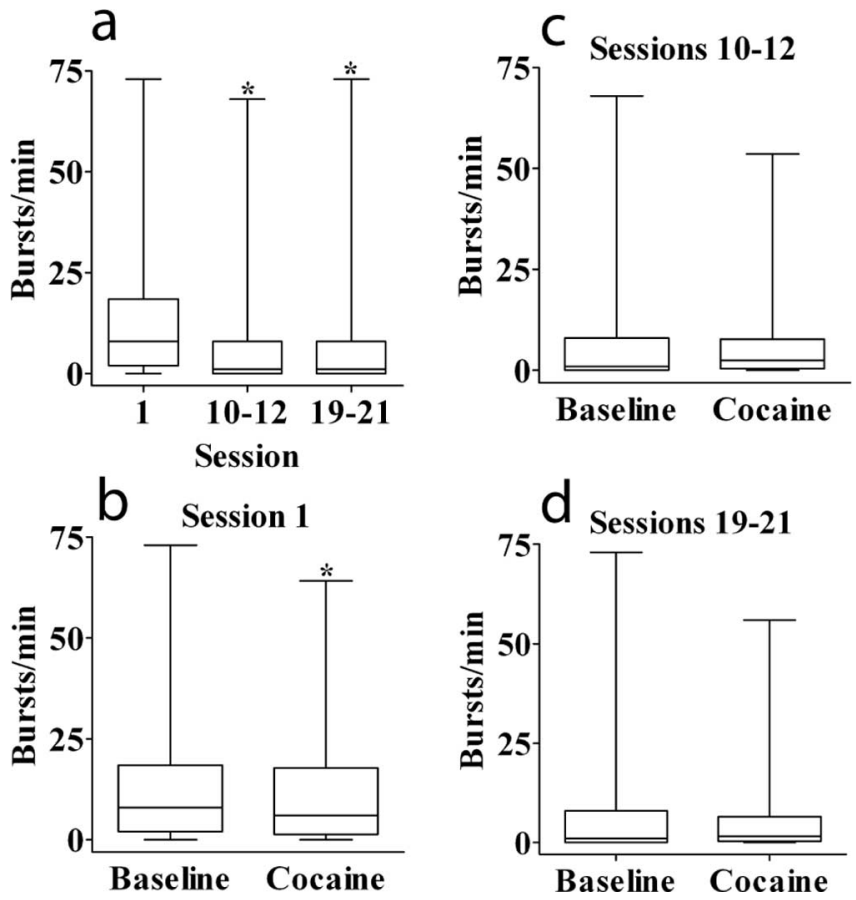

Figure 2. Effects of cocaine on the burst rate of PFC neurons. Data are as in Figure 1. $\boldsymbol{a}, \boldsymbol{b}$, Significantly different (Kruskal-Wallis test, ${ }^{*} p<0.0001$ ) from the basal burst rate recorded from session $1(\boldsymbol{a})$ and from the baseline rate during session $1(\boldsymbol{b}) . \boldsymbol{c}, \boldsymbol{d}$, Note that cocaine failed to decrease the burst rate after multiple SA sessions. Box-and-whisker graphs are presented as in Figure 1.

and 19-21 was $1.0(0.0-8.0)$ and $1.0(0.0-8.0)$ bursts/min in both cases, which was significantly lower than the session 1 rate of $8.0(2.0-18.5)$ bursts/min (Kruskal-Wallis test, $p<0.0001)$. No differences were found between sessions $10-12$ and 19-21. Cocaine significantly decreased the burst rate in the first SA session (Wilcoxon signed-rank test, $p<0.01$ ) but not in the other sessions.

We then studied the effects of chronic cocaine SA on burst strength, measured as the rate within a burst and burst duration. As shown in Figure 3, the median (50th percentile) basal rates within a burst in sessions $10-12$ and 19-21 were $15.0(0.0-21.5)$ and $15.0(0.0-20.2)$ spikes/s, respectively, which were significantly lower compared with session 1, 20.2 (15.6-24.5) spikes/s (Kruskal-Wallis test, $p<0.0001)$. There were no significant differences between sessions 10-12 and 19-21. Cocaine did not significantly decrease the rate within a burst during the first SA session but significantly increased it in sessions $10-12$ and 19-21 (Wilcoxon signed-rank test, $p<0.01$ and $p<0.001$, respectively).

The effect of cocaine on burst duration is summarized in Table 1. Again, basal burst duration was significantly decreased in sessions 10-12 and 19-21 relative to session 1 (Kruskal-Wallis test, $p<0.001$ ), and there was no significant difference between sessions 10-12 and 19-21. Moreover, cocaine did not decrease burst duration during the first SA session but significantly increased it in sessions 10-12 and 19-21 (Wilcoxon signed-rank test, $p<0.01$ and $p<0.05$, respectively).

\section{Discussion}

Our results demonstrate that PFC basal activity was significantly decreased after multiple weeks of cocaine SA training. In addition, the drug produced different effects on PFC activity in cocaine-naive and cocaine-experienced rats. Thus, during the 

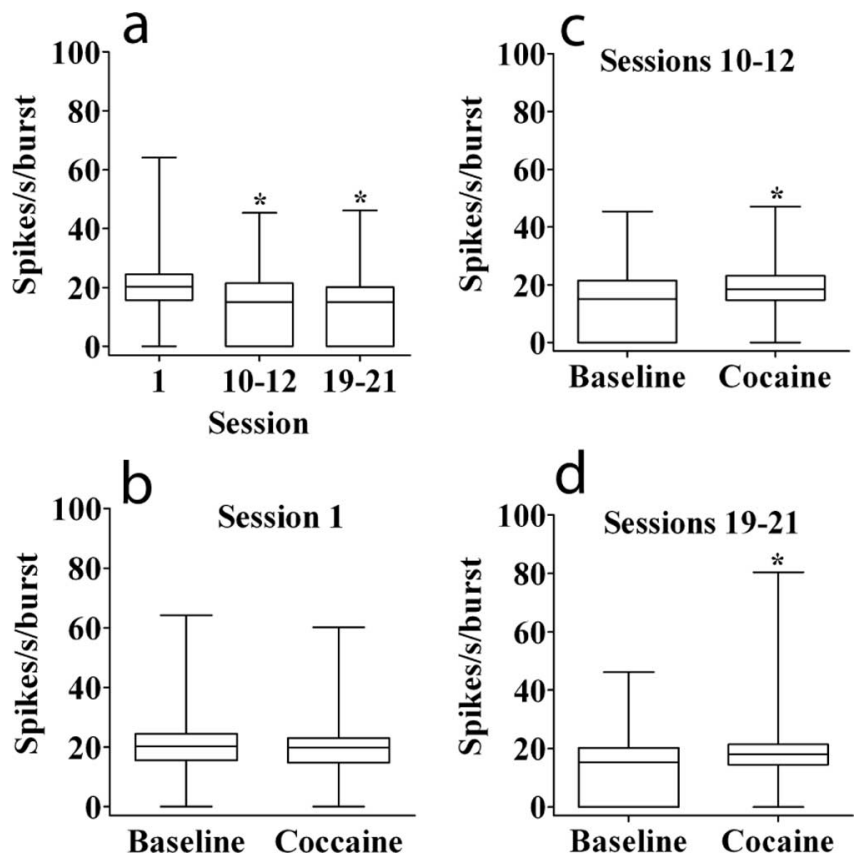

Figure 3. Effects of cocaine on the firing rate within a burst. Data are as in Figure 1. The asterisk indicates significant difference (Kruskal-Wallis test, $p<0.0001$ ) from the basal rate recorded from session $1(\boldsymbol{a})$ and from the baseline rate after multiple sessions $(\boldsymbol{c}, \boldsymbol{d})$ but not during session $1(\boldsymbol{b})$. Box-and-whisker graphs are presented as in Figure 1.

Table 1. Effects of cocaine on burst duration

\begin{tabular}{|c|c|c|}
\hline \multirow[b]{2}{*}{ Cocaine SA } & \multicolumn{2}{|c|}{ Median burst duration (ms) ${ }^{a}$} \\
\hline & Baseline & After cocaine \\
\hline Session 1 & $176.5(99.6-247.5)$ & $172.5(141.1-261.2)$ \\
\hline Sessions $10-12$ & $93.3(0.0-194.7)^{* *}$ & $165.6(123.8-193.5)^{*}$ \\
\hline Sessions 19-21 & $134.3(0.0-232.9)^{* *}$ & $163.6(111.6-225.6)^{*}$ \\
\hline
\end{tabular}

first cocaine SA session, rats responded to cocaine with decreased firing and burst activity, but these effects disappeared after $\sim 2$ and 3 weeks of cocaine SA. PFC neurons also showed increased burst strength in response to cocaine as evidenced by the increased firing rate within a burst and burst duration in cocaineexperienced but not cocaine-naive rats. Together, these data support the idea that although chronic cocaine exposure decreases PFC function, processing of cocaine-related information is enhanced. Such enhancement may contribute to increased control by cocaine over cocaine-seeking behavior.

One alternative explanation for increased burst strength is that cocaine may normalize PFC activity rather than increase processing of cocaine-related information. For example, basal burst strength was significantly decreased in sessions 19-21 compared with session 1, but cocaine appeared to bring basal burst strength back to the session 1 level. If nonspecific normalizing effects are responsible for this reversal, cocaine should also normalize the firing rate and burst rate. The fact that we did not observe such effects argues against this explanation.

The resting membrane potential of PFC pyramidal neurons alternates between an "up" state, when these neurons fire, and a "down" state, when they do not (Peters et al., 2000). In anesthetized rats, acute cocaine causes depolarization, eliminates the oscillation between down and up states, and decreases the firing rate of these neurons (Trantham-Davidson and Lavin, 2004). It is possible that elimination of membrane oscillation prevents PFC neurons from reaching the up state and thus is responsible for the decrease in firing rate. This interpretation is consistent with our results that self-administered cocaine decreased the firing rate in cocaine-naive rats. Similarly, chronic cocaine also depolarizes PFC neurons and eliminates the membrane oscillation; these effects, moreover, are still apparent 2 weeks after the last administration of cocaine (Trantham et al., 2002). The long-lasting membrane effects may explain the decreased basal firing rate observed in the current experiments.

Although cocaine decreases PFC activity in cocaine-naive rats, such inhibitory effects are no longer apparent in cocaineexperienced rats. In fact, in these rats, PFC neurons showed increased burst strength in response to cocaine. It has been demonstrated that repeated exposure to cocaine decreases inwardly rectifying $\mathrm{K}^{+}$currents and increases voltage-sensitive $\mathrm{Ca}^{2+}$ currents in response to depolarizing stimuli (Dong et al., 2005; Nasif et al., 2005). These changes could contribute to enhanced responsiveness of PFC neurons to psychostimulants. One potential mechanism underlying the effects of cocaine on $\mathrm{K}^{+}$channels involves the cAMP/protein kinase A (PKA) pathway (Dong et al., 2005). PKA can phosphorylate $\mathrm{K}^{+}$channels and consequently decrease $\mathrm{K}^{+}$currents (Dong and White, 2003). Repeated exposure to cocaine upregulates the cAMP/PKA pathway in PFC neurons (Dong et al., 2005). The upregulated PKA may increase the phosphorylation of $\mathrm{K}^{+}$channels and, consequently, decrease $\mathrm{K}^{+}$ currents. Both $\mathrm{D}_{1}$ - and $\mathrm{D}_{2}$-like dopamine (DA) receptors regulate the $\mathrm{CAMP} / \mathrm{PKA}$ pathway. Activation of $\mathrm{D}_{1}$-like receptors increases activity of the CAMP/PKA pathway, whereas activation of $\mathrm{D}_{2}$-like receptors decreases it (Stoof and Kebabian, 1981). Because cocaine increases extracellular DA levels by blocking DA uptake, it may either increase or decrease PKA activity depending on which receptor subtype is predominantly activated. There is evidence that chronic cocaine may preferentially increase signaling through $\mathrm{D}_{1}$-like receptors. For example, AGS3, a member of the activator-of-G-protein-signaling family, is upregulated in rat PFC by repeated exposure to cocaine (Bowers et al., 2004). This protein disrupts the interaction between $\mathrm{G}_{\mathrm{i} \alpha}$ and $\mathrm{G}_{\beta} \gamma$ subunits to decrease signal transduction related to $G_{i \alpha}$. Because $D_{2}$-like receptors are associated with $\mathrm{G}_{\mathrm{i} \alpha}$, upregulation of PFC ASG3 will decrease $\mathrm{D}_{2}$-like receptor-mediated signaling and therefore preferentially increase $D_{1}$-like receptor-mediated signaling. An increase in $\mathrm{D}_{1}$-like receptor-mediated signaling may contribute to upregulation of cAMP/PKA, a decrease in $\mathrm{K}^{+}$currents, and thus increased responsiveness of PFC neurons to cocaine.

DA plays a critical role in sustaining activation of primate dorsolateral PFC neurons during a delayed-response task (Sawaguchi, 2001); this persistent activation has been proposed as a neuronal mechanism involved in working memory (Goldman-Rakic, 1995). The activity-sustaining effects of DA may be related to its effects on the membrane potential of PFC neurons. For example, burst stimulation of the ventral tegmental area induces up states of PFC neurons, and duration of the up states seems to be dependent on activation of $D_{1}$-like receptors (Lewis and O'Donnell, 2000). Thus, in cocaine-experienced rats, increased $\mathrm{D}_{1}$-like receptor signaling could increase residence time of the up state of PFC neurons. This increased up state could explain the increased burst duration induced by selfadministered cocaine in cocaine-experienced rats.

There is evidence that increased signaling through $\mathrm{D}_{1}$-like receptors in the PFC is critically involved in cocaine-seeking behavior. For example, overexpression of ASG3 increases cocaine- 
seeking behavior, whereas inhibition of ASG3 upregulation with antisense oligonucleotides microinfused into the PFC blocks reinstatement of cocaine-seeking behavior (Bowers et al., 2004). We recently demonstrated that blockade of $D_{1}$-like receptors in the PFC dose-dependently blocks cocaine-primed reinstatement of cocaine-seeking behavior (Sun and Rebec, 2005). Together, these data indicate that increased $\mathrm{D}_{1}$-like receptor-mediated signaling in the PFC after chronic exposure to cocaine may be an important mechanism underlying compulsive cocaine-seeking behavior.

In summary, our results provide direct evidence that chronic exposure to cocaine decreases basal activity in the PFC. Processing cocaine-related information, however, seems to be preferentially increased as evidenced by increased burst strength in response to self-administered cocaine. The combination of lower basal activity and enhanced burst strength may bias the PFC to process information related to cocaine and thereby contribute to focused and persistent cocaine-related behavior.

\section{References}

Bowers MS, McFarland K, Lake RW, Peterson YK, Lapish CC, Gregory ML, Lanier SM, Kalivas PW (2004) Activator of G protein signaling 3: a gatekeeper of cocaine sensitization and drug seeking. Neuron 42:269-281.

Childress AR, Mozley PD, McElgin W, Fitzgerald J, Reivich M, O'Brien CP (1999) Limbic activation during cue-induced cocaine craving. Am J Psychiatry 156:11-18.

Dias R, Robbins TW, Roberts AC (1996) Primate analogue of the Wisconsin Card Sorting Test: effects of excitotoxic lesions of the prefrontal cortex in the marmoset. Behav Neurosci 110:872-886.

Dong Y, White FJ (2003) Dopamine $\mathrm{D}_{1}$-class receptors selectively modulate a slowly inactivating potassium current in rat medial prefrontal cortex pyramidal neurons. J Neurosci 23:2686-2695.

Dong Y, Nasif FJ, Tsui JJ, Ju WY, Cooper DC, Hu XT, Malenka RC, White FJ (2005) Cocaine-induced plasticity of intrinsic membrane properties in prefrontal cortex pyramidal neurons: adaptations in potassium currents. J Neurosci 25:936-940.

Goldman-Rakic PS (1995) Cellular basis of working memory. Neuron 14:477-485.

Gulley JM, Reed JL, Kuwajima M, Rebec GV (2004) Amphetamine-induced behavioral activation is associated with variable changes in basal ganglia output neurons recorded from awake, behaving rats. Brain Res 1012:108-118.

Jentsch JD, Taylor JR (1999) Impulsivity resulting from frontostriatal dysfunction in drug abuse: implications for the control of behavior by reward-related stimuli. Psychopharmacology 146:373-390.

Jentsch JD, Olausson P, De La Garza II R, Taylor JR (2002) Impairments of reversal learning and response perseveration after repeated, intermittent cocaine administrations to monkeys. Neuropsychopharmacology 26:183-190.

Laviolette SR, Lipski WJ, Grace AA, Laviolette SR, Lipski WJ, Grace AA (2005) A subpopulation of neurons in the medial prefrontal cortex encodes emotional learning with burst and frequency codes through a dopamine $\mathrm{D}_{4}$ receptor-dependent basolateral amygdala input. J Neurosci 25:6066-6075.

Legendy CR, Salcman M, Legendy CR, Salcman M (1985) Bursts and recurrences of bursts in the spike trains of spontaneously active striate cortex neurons. J Neurophysiol 53:926-939.

Lewis BL, O’Donnell P (2000) Ventral tegmental area afferents to the prefrontal cortex maintain membrane potential "up" states in pyramidal neurons via $\mathrm{D}(1)$ dopamine receptors. Cereb Cortex 10:1168-1175.

Nasif FJ, Hu XT, White FJ (2005) Repeated cocaine administration increases voltage-sensitive calcium currents in response to membrane depolarization in medial prefrontal cortex pyramidal neurons. J Neurosci 25:3674-3679.

Paxinos G, Watson C (1998) The rat brain in stereotaxic coordinates. New York: Academic.

Peters YM, Lewis BL, O’Donnell P (2000) Synchronous activity in the ventral tegmental area and prefrontal cortex. Ann NY Acad Sci 909:267-269.

Quirk MC, Wilson MA, Quirk MC, Wilson MA (1999) Interaction between spike waveform classification and temporal sequence detection. J Neurosci Methods [Erratum (2000) 99:143] 94:41-52.

Rebec GV, Sun W (2005) Neuronal substrates of relapse to cocaine-seeking behavior: role of prefrontal cortex. J Exp Anal Behav 84:653-666.

Roberts AC, Wallis JD (2000) Inhibitory control and affective processing in the prefrontal cortex: neuropsychological studies in the common marmoset. Cereb Cortex 10:252-262.

Sawaguchi T (2001) The effects of dopamine and its antagonists on directional delay-period activity of prefrontal neurons in monkeys during an oculomotor delayed-response task. Neurosci Res 41:115-128.

Stoof JC, Kebabian JW (1981) Opposing roles for D-1 and D-2 dopamine receptors in efflux of cyclic AMP from rat neostriatum. Nature 294:366-368.

Sun W, Rebec GV (2003) Lidocaine inactivation of ventral subiculum attenuates cocaine-seeking behavior in rats. J Neurosci 23:10258-10264.

Sun W, Rebec GV (2005) The role of prefrontal cortex D1-like and D2-like receptors in cocaine-seeking behavior in rats. Psychopharmacology (Berl) 177:315-323.

Trantham-Davidson H, Lavin A (2004) Acute cocaine administration depresses cortical activity. Neuropsychopharmacology 29:2046-2051.

Trantham H, Szumlinski KK, McFarland K, Kalivas PW, Lavin A (2002) Repeated cocaine administration alters the electrophysiological properties of prefrontal cortical neurons. Neuroscience 113:749-753.

Volkow ND, Fowler JS, Wang GJ (2003) The addicted human brain: insights from imaging studies. J Clin Invest 111:1444-1451. 